06

\title{
Исследование влияния постоянного электрического поля на диэлектрические свойства $\mathrm{LiNbO}_{3}$
}

\author{
(C) А.В. Яценко, ${ }^{1}$ С.М. Кострицкий ${ }^{2}$ \\ ${ }^{1}$ Крымский федеральный университет им. В.И. Вернадского, \\ 95007 Симферополь, Россия \\ ${ }^{2}$ НПК Оптолинк, \\ 124489 Зеленоград, Москва, Россия \\ e-mail: yatsenkoav@cfuv.ru
}

Поступило в Редакцию 31 января 2019 г.

В окончательной редакции 2 апреля 2019 г.

Принято к публикации 1 ноября 2019 г.

\begin{abstract}
Экспериментально исследовано влияние сильного электрического поля на диэлектрические свойства номинально беспримесных кристаллов $\mathrm{LiNbO}_{3}$ конгруэнтного состава в области низких частот. Показано, что внешнее постоянное электрическое поле, направленное вдоль или антипараллельно полярной оси кристалла, а также поле пироэлектрической природы при напряженности поля до $14 \mathrm{\kappa V} / \mathrm{cm}$, в пределах погрешности не влияют на диэлектрические свойства кристалла в области низких частот.
\end{abstract}

Ключевые слова: ниобат лития, пироэлектрический эффект, диэлектрические свойства, электрооптический эффект.

DOI: $10.21883 / J T F .2020 .04 .49090 .29-19$

Кристалл ниобата лития (НЛ) $\mathrm{LiNbO}_{3}$ в настоящее время является одним из наиболее практически востребованных сегнетоэлектрических материалов [1]. Именно НЛ и изоструктурный ему танталат лития $\mathrm{LiTaO}_{3}$ наиболее часто применяется для изготовления устройств на поверхностных акустических волнах (ПАВ) и в оптоэлектронике. При использовании изделий на основе НЛ важное значение приобретает прогнозируемость влияния внешних факторов (особенно температуры) на их параметры. Так как кристаллы НЛ обладают ярко выраженными пироэлектрическими свойствами, изменение температуры сопровождается возникновением дополнительного электрического поля как внутри, так и снаружи кристалла, которое может существенно влиять на эксплуатационные свойства изделия [2,3]. Поэтому вопрос о влиянии сильного электрического поля на принципиальные для работы этих устройств материальные параметры НЛ [4] является весьма принципиальным.

Известно, что в модуляторах лазерного излучения, выполненных на пластинах $x$ и $z$ срезов НЛ, поле пироэлектрической природы сильно влияет на качество работы самого модулятора $[3,5]$ и приводит к нестабильности лазерных гироскопов на их основе [6]. Кроме известных механизмов возникновения нестабильности работы модуляторов $[3,5]$ гипотетически можно рассматривать еще один - возможное влияние полей пироэлектрической природы на статические диэлектрические свойства НЛ. В модуляторах с тонким буферным слоем из $\mathrm{SiO}_{2}$ такой эффект должен приводить к изменению структуры электрического поля между управляющими электродами и соответственно изменению разности хода световых пучков при фиксированном управляющем напряжении.
Очевидно, что возможное изменение значений главных компонент тензора диэлектрической проницаемости НЛ должно привести и к изменению параметров изделия на ПАВ [3].

В доступной нам литературе отсутствует информация о результатах прямых экспериментов по исследованию влияния сильного электрического поля на статические диэлектрические свойства кристаллов НЛ, поэтому о состоянии проблемы можно судить только по косвенным данным.

Известно [7], что приложение вдоль полярного направления кристаллов $\mathrm{LiNbO}_{3}$ постоянного электрического поля $E_{z}$ приводит к смещению катионов относительно кислородной подрешетки. Согласно [7], при $E_{z}=51.5 \mathrm{kV} / \mathrm{cm}$ смещение ионов $\mathrm{Nb}\left(\Delta z_{\mathrm{Nb}}\right)$ и $\mathrm{Li}\left(\Delta z_{\mathrm{Li}}\right)$ составляет $4.9 \cdot 10^{-4}$ и $13 \cdot 10^{-4} \AA$ соответственно. Это позволяет оценить изменение упругого (ионного) вклада в поляризацию $\Delta P$ :

$$
\Delta P=\frac{6\left(q_{\mathrm{Nb}} \Delta z_{\mathrm{Nb}}+q_{\mathrm{Li}} \Delta z_{\mathrm{Li}}\right)}{V}
$$

где $q_{\mathrm{Nb}}$ и $q_{\mathrm{Li}}-$ эффективные заряды ионов $\mathrm{Nb}$ и $\mathrm{Li}, V-$ объем элементарной ячейки кристалла.

Используя (1), проведем оценку значения компоненты тензора диэлектрической проницаемости вдоль полярной оси $\varepsilon_{33}$ :

$$
\varepsilon_{33}=\frac{\Delta P}{\varepsilon_{0} E_{z}}+1,
$$

где $\varepsilon_{0}-$ электрическая постоянная. Полагая, что $q_{\mathrm{Nb}}=3.376|e|$ и $q_{\mathrm{Li}}=0.98|e|$, где $|e|-$ модуль заряда электрона $[8]$, получим: $\varepsilon_{33} \cong 20.4$. Как известно, ионный 
вклад в $\varepsilon_{33}$ для кристаллов НЛ является доминирующим [9], однако даже если положить значение $q_{\mathrm{Nb}}$ равным формальному заряду $+5|e|$, то расчетное значение $\varepsilon_{33}$ будет существенно меньше реального: $\varepsilon_{33} \cong 29$ для номинально беспримесного кристалла НЛ конгруэнтного состава [10]. Следовательно, можно предполагать, что приложение к кристаллам НЛ постоянного электрического поля может привести к проявлению полевой зависимости компонент тензора статической диэлектрической проницаемости. Косвенное подтверждение существования такого эффекта приводится в [11].

Исследование влияния одноосного давления на емкость конденсатора, изготовленного из НЛ с исходной емкостью $C_{0}$, показало, что при давлении $0.1 \mathrm{GPa}$ относительное изменение емкости $\Delta C / C_{0}$ составляет $0.8 \%$ [12]. Пьезоэлектрический коэффициент НЛ $d_{33}=6.2 \mathrm{pC} / \mathrm{N}$ [13], поэтому такому значению давления должно соответствовать пьезоиндуцированное электрическое поле $E_{z} \approx 24 \mathrm{kV} / \mathrm{cm}$. Однако при изучении влияния гидростатического сжатия на значение $\varepsilon_{33}$ (с учетом изменения размеров образца) [14] при давлении вплоть до $1 \mathrm{GPa}$, было установлено, что относительное изменение $\varepsilon_{33}$ составило всего $0.07 \%$.

Основной целью настоящей работы является исследование возможного влияния сильного постоянного электрического поля на диэлектрические свойства кристаллов НЛ конгруэнтного состава в области низких частот.

\section{Образец и методика измерения}

Для исследования влияния постоянного электрического поля на диэлектрические свойства НЛ была использована заготовка для изготовления оптического модулятора (фирма „Оптолинк“, г. Зеленоград). Эта заготовка представляет собой тонкую $(1.00 \mathrm{~mm})$ пластинку неполярного среза кристалла НЛ конгруэнтного состава (коммерческий продукт фирмы Jamaju Ceramics Company), на которой сформированы 5 электродов. Конфигурация электродов изображена на рис. 1.

Ширина чипа составляет $3.45 \mathrm{~mm}$, общая ширина группы электродов $1.86 \mathrm{~mm}$, расстояния от электродов до краев чипа $0.80 \mathrm{~mm}$. Зазоры между электродами составляют $14 \mu \mathrm{m}$.

Полярные грани образца были прошлифованы корундовым порошком и на их поверхность нанесена индийгаллиевая эвтектика. Эти дополнительные электроды

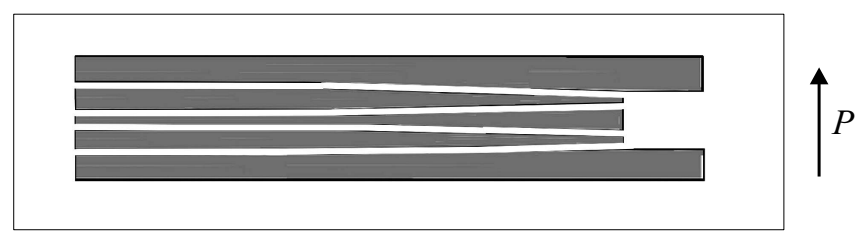

Рис. 1. Топология расположения электродов на поверхности чипа. Стрелкой указано направление вектора спонтанной поляризации $P$.

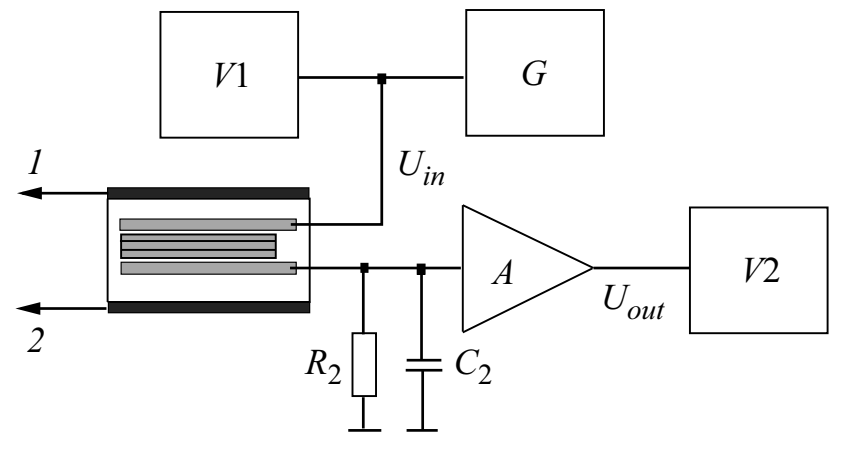

Рис. 2. Блок-схема измерительной части установки. $A-$ усилитель, $G$ - генератор синусоидального напряжения, $V 1$ и $V 2$ - вольтметры, 1 и 2 - дополнительные электроды, нанесенные на полярные поверхности чипа.

предназначены для управления состоянием кристалла в процессе выполнения измерений.

Блок-схема экспериментальной установки для исследования межэлектродной емкости чипа приведена на рис. 2.

В качестве источника синусоидального напряжения $(G)$ применен генератор напряжения специальной формы типа Г6-28 с дополнительным усилителем напряжения. В состав устройства входит широкополосный усилитель с дискретно изменяемым коэффициентом усиления $K_{0}$. Выходное напряжение генератора и напряжение на выходе усилителя измерялось прецизионными вольтметрами типа В7-78/1. Исследуемый образец на специальном керамическом держателе находится в экранированной измерительной головке, которая, в свою очередь, размещается внутри нагревательного модуля. Воздух внутри нагревательного модуля осушался силикагелем, а температура измерялась дифференциальной термопарой хромель-алюмель.

Несложно показать, что модуль коэффициента передачи $|K(\omega)|$ цепи, состоящей из исследуемого чипа и усилителя при условии, что активное сопротивление чипа много больше входного сопротивления усилителя $R_{2}$ на угловой частоте $\omega$ описывается следующим выражением:

$$
|K(\omega)|=\frac{\omega C_{1} R_{2} K_{0}}{\sqrt{1+\omega^{2} R_{2}^{2}\left(C_{1}+C_{2}\right)^{2}}}=\frac{U_{\text {out }}}{U_{\text {in }}}
$$

где $U_{\text {out }}$ - амплитуда выходного напряжения усилителя, $U_{i n}$ - выходное напряжение генератора, $C_{1}-$ емкость чипа, $C_{2}-$ входная емкость усилителя, $R_{2}-$ входное сопротивление усилителя. Таким образом, экспериментально измеряя частотную зависимость $|K(\omega)|$ при известных значениях $R_{2}$ и $C_{2}$ при помощи выражения (3), можно установить частотную зависимость $C_{1}$. 


\section{Экспериментальные результаты и обсуждение}

На первом этапе при помощи установки, использовавшейся ранее [15], была исследована температурная зависимость сопротивления чипа $R_{1}$ при приложении к электродам постоянного напряжения. Соответствующие результаты приводятся на рис. 3. Видно, что во всем диапазоне температур значение $R_{1}$ на много порядков превышает использованное нами значение $R_{2}=10 \mathrm{k} \Omega$, что позволяет использовать для расчета емкости чипа $C_{1}$ выражение (3).

Суммарный (не металлизированный) зазор между измерительными электродами составляет $56 \mu \mathrm{m}$, поэтому при приложении к ним разности потенциалов $5.6 \mathrm{~V}$ оценочное максимальное значение напряженности электрического поля в кристалле составит $E \approx 1 \mathrm{kV} / \mathrm{cm}$. Это дает возможность исследования вольт-фарадной зависимости исследуемого чипа в достаточно широком диапазоне значений $E$. В качестве точек подключения были выбраны внешние электроды основной группы, нанесенные на неполярную поверхность чипа (рис. 2). Результаты измерений для ряда фиксированных частот, выполненных при комнатной температуре, представлены на рис. 4. Так же было установлено, что для диапазона частот $(0.04-300) \mathrm{kHz}$ дисперсия диэлектрической проницаемости исследованного чипа в рамках погрешности измерения не проявляется.

Непосредственно влияние внешнего электрического поля на диэлектрические свойства исследуемого чипа исследовалось двумя методами.

В первом эксперименте на электроды, нанесенные на полярные грани чипа, от специального биполярного источника подавалось медленно меняющееся напряжение, так что максимальное значение разности потенциалов между полярными гранями составляло $4 \mathrm{kV}$. Это было бы эквивалентно напряженности поля $E_{z}=11.6 \mathrm{kV} / \mathrm{cm}$

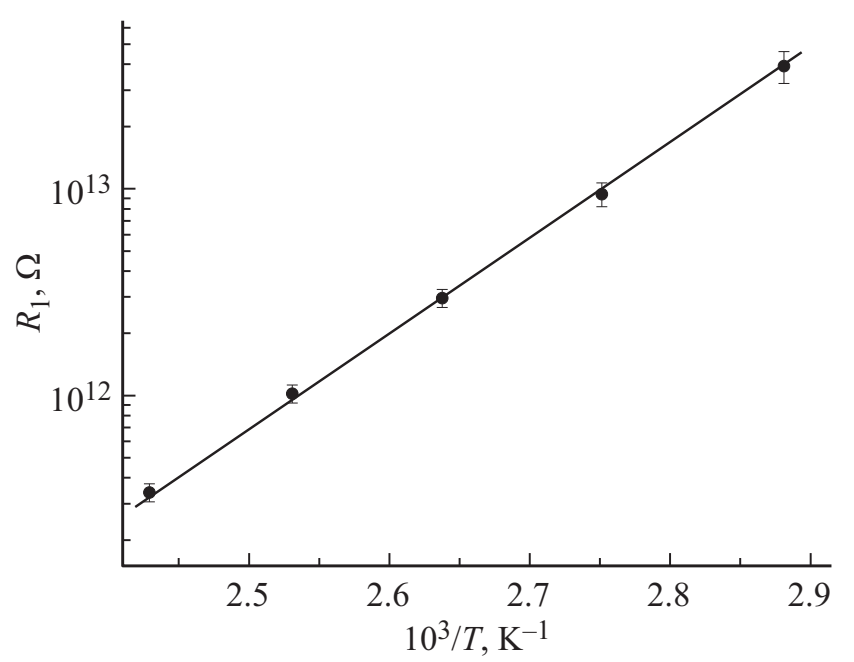

Рис. 3. Температурная зависимость межэлектродного сопротивления $R_{1}$.

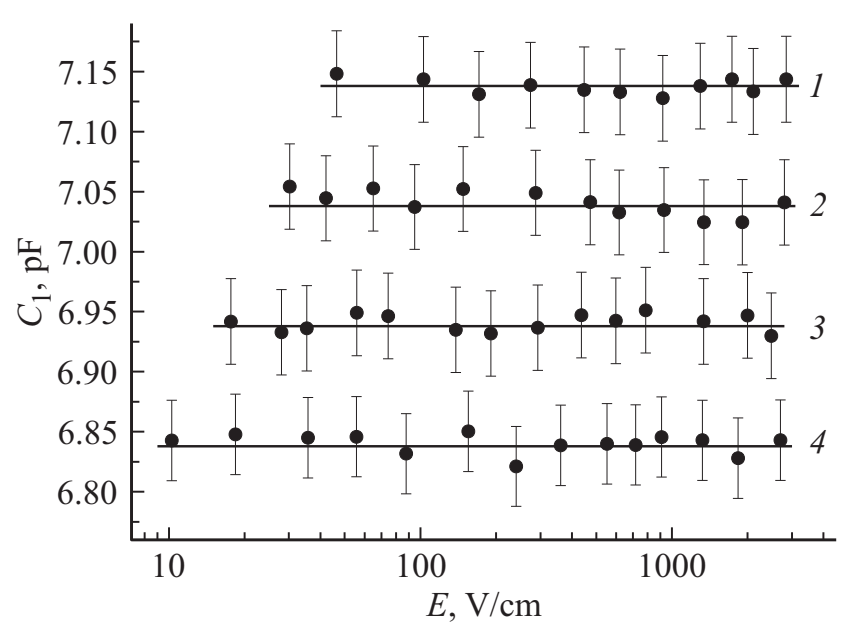

Рис. 4. Вольт-фарадные зависимости емкости исследуемого чипа, полученные на частотах 1 (1), 2 (2), 4 (3) и $8 \mathrm{kHz}(4)$. Для наглядности данные для последующих частот сдвинуты друг относительно друга на $0.10 \mathrm{pF}$ вниз.

внутри чипа таких же размеров, но без электродов на поверхности. Измерения емкости $C_{1}$ проводились на частотах $0.1,1.0$ и $10 \mathrm{kHz}$ при фиксированной (комнатной) температуре. Изменения емкости $C_{1}$ в процессе нарастания разности потенциалов между дополнительными электродами в пределах точности эксперимента не наблюдалось.

Так как НЛ имеет ярко выраженные пироэлектрические свойства, при изменении температуры чипа на величину $\Delta T$ на полярных поверхностях появляются некомпенсированные заряды с абсолютным значениeм $\Delta Q$ :

$$
\Delta Q=\gamma \Delta T S
$$

где $S$ - площадь полярной грани, $\gamma$ - пироэлектрический коэффициент.

Специально проведенное исследование пироэлектрических свойств данного чипа и второго кристалла НЛ этой же фирмы по методике, использованной в [16], показало, что для этих образцов значение $\gamma \cong 100 \mu \mathrm{C} \cdot\left(\mathrm{m}^{2} \cdot \mathrm{K}\right)^{-1}$.

Некомпенсированные заряды, локализованные на полярных поверхностях чипа, приводят к возникновению дополнительного поля, антипараллельного полярной оси кристалла $E_{p}$ :

$$
E_{p}=\frac{\gamma \Delta T}{\varepsilon_{33} \varepsilon_{0}}
$$

Принимая во внимание, что для данного чипа $\gamma \cong 100 \mu \mathrm{C} \cdot\left(\mathrm{m}^{2} \cdot \mathrm{K}\right)^{-1}$ и $\varepsilon_{33} \cong 29$, при $\Delta T=1 \mathrm{~K}$ значение $E_{p}$ составит величину $3.7 \mathrm{kV} / \mathrm{cm}$. Эта оценка справедлива при условии, что в процессе разогрева избыточные заряды не стекают с полярных поверхностей. Реально стекание избыточных зарядов может происходить либо за счет собственной объемной проводимости кристалла, либо по поверхности кристалла в виде микропробоев, 
либо за счет ионизации окружающего воздуха быстрыми электронами [17] и ограничивает максимального значения $E_{\max }$ дополнительного внутрикристаллического поля.

Для оценки $E_{\max }$ была проведена серия экспериментов по исследованию электрического отклика (разность потенциалов $U$ между двумя внешними электродами модулятора при нагрузочном сопротивлении $20 \mathrm{M} \Omega$ ) таких же чипов на изменение температуры. Типичный отклик, зарегистрированный двухкоординатным потенциометром Н307/2, приведен на рис. 5. Было установлено, что при разогреве в атмосфере сухого воздуха чипа с обезжиренной и обезвоженной поверхностью первое скачкообразное изменение заряда, происходит при $\Delta T=(4-6) \mathrm{K}$. Этому отвечает значение $E_{\max }=(14.8-22.2) \mathrm{kV} / \mathrm{cm}$.

При электрическом объединении дополнительных электродов на полярных поверхностях при разогреве чипа избыточные заряды компенсируют друг друга, и дополнительное поле $E_{p}$ не возникает. Сравнивая температурную зависимость межэлектродной емкости $C_{1}$ „свободного“ чипа и чипа с замкнутыми дополнительными электродами, можно оценить эффект влияния
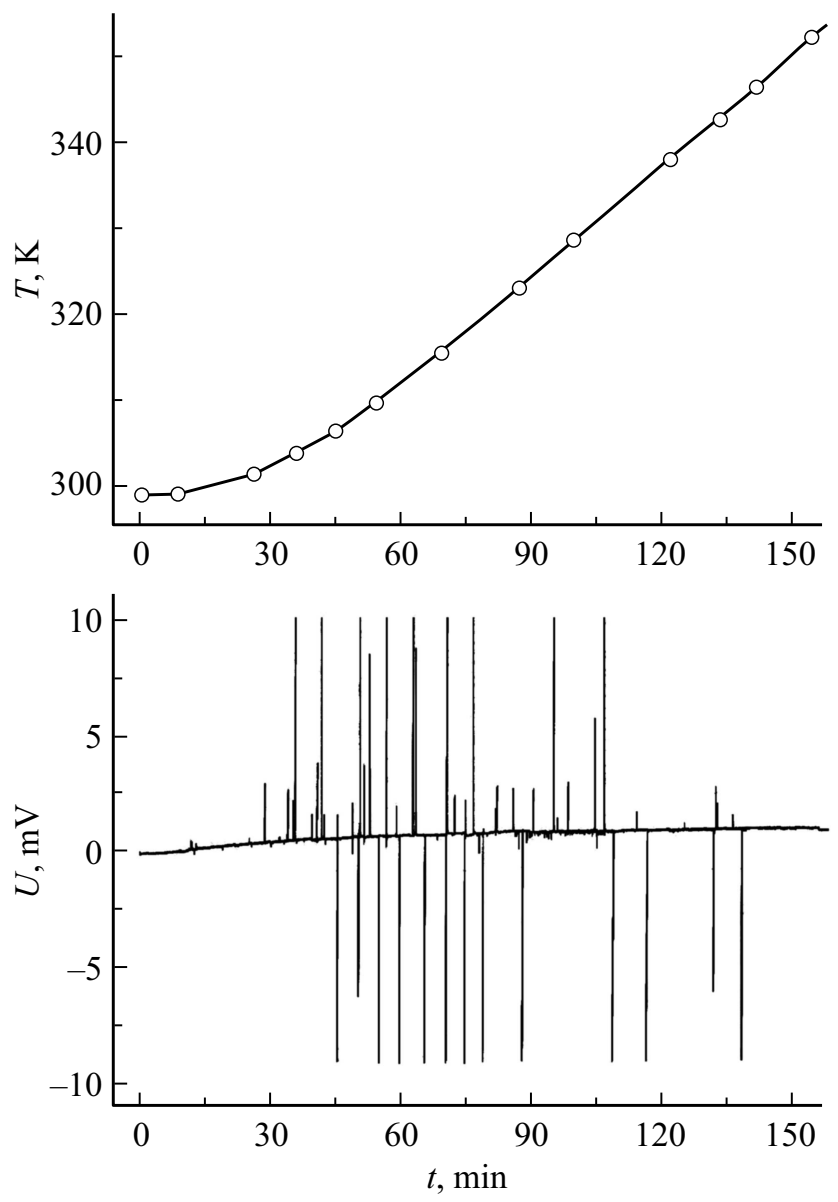

Рис. 5. Типичная электрическая реакция исследуемого чипа на изменение температуры. Вверху представлен временной алгоритм изменения температуры чипа.

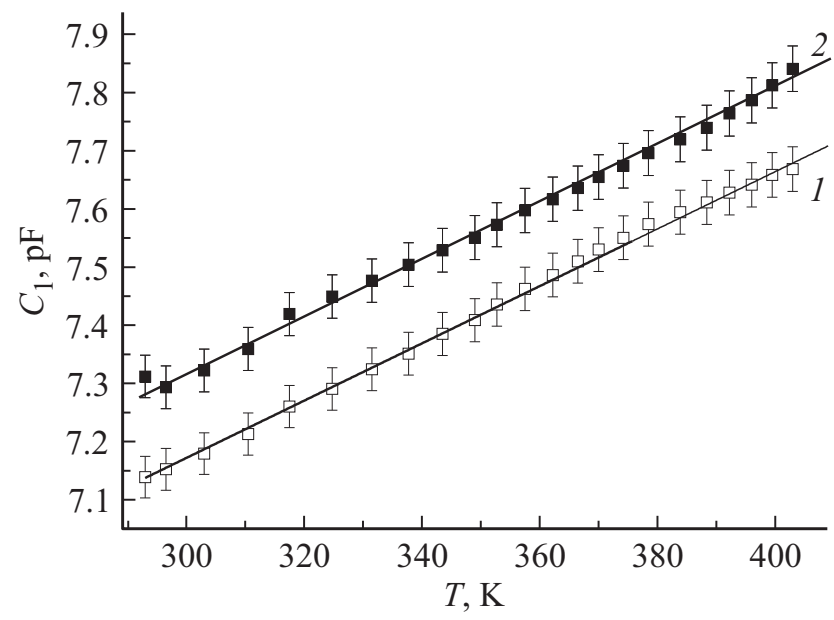

Рис. 6. Температурная зависимость емкости $C 1$ „свободного“ (1) и закороченного (2) чипов.

электрического поля на диэлектрические свойства чипа. Соответствующие экспериментальные данные, полученные на частоте $10 \mathrm{kHz}$ при одном и том же алгоритме разогрева кристалла (максимальная скорость нагрева $1.8 \mathrm{~K} / \mathrm{m})$, приводятся на рис. 6 .

Видно, что обе зависимости не имеют очевидных аномалий и в пределах погрешности хорошо апроксимируются полиномом первой степени. Небольшой сдвиг зависимостей друг относительно друга вызван частичной перестройкой структуры переменного электрического поля в приэлектродной области при соединении между собой дополнительных электродов, нанесенных на полярные поверхности чипа.

Аналогичные эксперименты были проведены на частотах 0.1, 1.0 и $100 \mathrm{kHz}$. При этом были получены подобные результаты, а какие-либо аномалии не наблюдались.

\section{Заключение}

Так как электрическое поле внутри исследованного чипа, возникающее при приложении разности потенциалов к двум крайним электродам модулятора, имеет достаточно сложную структуру, то измеряемая в данных экспериментах межэлектродная емкость $C_{1}$ при заданной конфигурации электродов зависит от всех компонент тензора диэлектрической проницаемости кристалла НЛ $\varepsilon_{i j}$. В случае обнаружения влияния постоянного электрического поля на значение $C_{1}$ соответствующие эффекты было бы невозможно разделить. Однако наши результаты однозначно свидетельствуют о том, что постоянное электрическое поле с напряженностью до $14 \mathrm{kV} / \mathrm{cm}$ в пределах погрешности не влияет на диэлектрические свойства кристаллов НЛ в области низких частот. 


\section{Конфликт интересов}

Авторы заявляют, что у них нет конфликта интересов.

\section{Список литературы}

[1] Arizmendi L. // Phys. Stat. Sol. A. 2004. Vol. 201. P. 253-283. DOI: $10.1002 /$ pssa.200303911

[2] Bordui P.F., Jundt D.H., Standifer E.M., Norwood R.G., Sawin R.L., Galipeau J.D. // J. Appl. Phys. 1999. Vol. 85. P. 3766-3769.

[3] Wooten E.J., Kissa K.M., Yi-Yan A., Murphy E.J. et al. // IEEE J. Selected Topics in Quantum Electronics. 2000. Vol. 6. P. 69-82.

[4] Kushibiki J., Takanaga I., Arakawa M., Sannomiya T. // IEEE Transactions of Ultrasonic, Ferroelectrics and Frequency Control. 1999. Vol. 46. P. 1315-1323.

[5] Nagata H., Li Y., Bosenberg W.R., Reiff G.L. // IEEE Photonics Techn. Lett. 2004. Vol. 16. P. 2233-2235.

[6] Zhang H., Liu J., Lin J., Ii W., Xue X., Huang A., Xiao J. // Appl. Phys. A. 2016. Vol. 122. 501. N 1-6.

DOI: $10.1007 / \mathrm{s} 00339-016-0008-8$

[7] Fujimoto I. // Acta Cryst. A. 1982. Vol. 38. P. 337-345.

[8] Яценко А.В. // Кристаллография. 2003. Т. 48. № 2. С. $343-$ 346. [Yatsenko A.V. // Cryst. Reports. 2003. Vol. 48. N 3. P. 500-503.]

[9] Cochard C., Spielmann T., Bahlawane N., Halpin A., Granzow T. // J. Phys. D: Appl. Phys. 2017. Vol. 50. 36LT01 (1-4). DOI: 10.1088 /136-6463/aa80b8

[10] Volk T.R. Wöhlecke M. Lithium Niobate. Defects, photorefraction and ferroelectric switching. Berlin: SpringerVerlag, 2008. $250 \mathrm{p}$.

[11] Ан Ф.М., Тхыонг Н.Х., Бурханов А.И., Медников С.В. // Бюлл. РАН. Физика. 2013. Т. 77. № 8. С. 1163-1165. [An P.M., Thuong N.H., Burkhanov A.I., Mednikov S.V. // Bull. RAS. Physics. 2013. Vol. 77. P. 1056-1058. DOI: 10.3103/S106287381308008X]

[12] Cho Y., Yamanouchi K. // J. Appl. Phys. 1987. Vol. 63. P. 875887.

[13] Yue W., Yi-Jian J. // Opt. Mat. 2003. Vol. 23. P. 403-408. DOI: $10.1016 / \mathrm{S} 0925-3467(02) 00328-2$

[14] Samara G.A. // Ferroelectrics. 1987. Vol. 73. P. 145-159.

[15] Ященко А.В., Палатников М.Н., Сидоров Н.В., Притуленко А.С., Евдокимов С.В. // ФТТ. 2015. Т. 57. Вып. 5. C. 932-936. [Yatsenko A.V., Palatnikov M.N., Sidorov N.V., Pritulenko A.S., Yevdokimov S.V. // Phys. Solid State. 2015. Vol. 57. N 8. P. $1547-1550$.

DOI: $10.1134 / \mathrm{S} 106378341505339]$

[16] Яценко А.В., Евдокимов С.В., Притуленко А.С., Сугак Д.Ю., Сольский И.М. // ФТТ. 2012. Т. 54. Вып. 11. С. 2095-2099. [Yatsenko A.V., Yevdokimov S.V., Pritulenko A.S., Sugak D.Yu., Solskii I.M. // Phys. Solid State. 2012. Vol. 54. P. 2231-2235. DOI: $10.1134 / \mathrm{S} 1063783412110339]$

[17] Rosenman G., Shur D., Krasik Ya.E., Dunaevsky A. // J. Appl. Phys. 2000. Vol. 88. P. 6109-6161. 